\title{
Extremely Dense Gamma-Ray Pulses in Electron Beam-Multifoil Collisions
}

\author{
Archana Sampath, ${ }^{1}$ Xavier Davoine, ${ }^{2,3}$ Sébastien Corde, ${ }^{4}$ Laurent Gremillet, ${ }^{2,3}$ Max Gilljohann, ${ }^{4}$ Maitreyi Sangal, ${ }^{1}$ \\ Christoph H. Keitel, ${ }^{1}$ Robert Ariniello, ${ }^{5}$ John Cary, ${ }^{5}$ Henrik Ekerfelt, ${ }^{6}$ Claudio Emma, ${ }^{6}$ Frederico Fiuza, ${ }^{6}$ Hiroki Fujii, ${ }^{7}$ \\ Mark Hogan, ${ }^{6}$ Chan Joshi, ${ }^{7}$ Alexander Knetsch, ${ }^{4}$ Olena Kononenko, ${ }^{4}$ Valentina Lee, ${ }^{5}$ Mike Litos, ${ }^{5}$ Kenneth Marsh, ${ }^{7}$ \\ Zan Nie, ${ }^{7}$ Brendan O'Shea, ${ }^{6}$ J. Ryan Peterson, ${ }^{6,8}$ Pablo San Miguel Claveria, ${ }^{4}$ Doug Storey, ${ }^{6}$ Yipeng Wu, ${ }^{7}$ Xinlu Xu, ${ }^{6}$ \\ Chaojie Zhang, ${ }^{7}$ and Matteo Tamburini $\oplus^{1, *}$ \\ ${ }^{1}$ Max-Planck-Institut für Kernphysik, Saupfercheckweg 1, D-69117 Heidelberg, Germany \\ ${ }^{2}$ CEA, DAM, DIF, 91297 Arpajon, France \\ ${ }^{3}$ Université Paris-Saclay, CEA, LMCE, 91680 Bruyères-le-Châtel, France \\ ${ }^{4}$ LOA, ENSTA Paris, CNRS, Ecole Polytechnique, Institut Polytechnique de Paris, 91762 Palaiseau, France \\ ${ }^{5}$ University of Colorado Boulder, Department of Physics, Center for Integrated Plasma Studies, \\ Boulder, Colorado 80309, USA \\ ${ }^{6}$ SLAC National Accelerator Laboratory, Menlo Park, California 94025, USA \\ ${ }^{7}$ University of California Los Angeles, Los Angeles, California 90095, USA \\ ${ }^{8}$ Stanford University, Physics Department, Stanford, California 94305, USA
}

(Received 25 September 2020; revised 18 December 2020; accepted 8 January 2021; published 12 February 2021)

\begin{abstract}
Sources of high-energy photons have important applications in almost all areas of research. However, the photon flux and intensity of existing sources is strongly limited for photon energies above a few hundred $\mathrm{keV}$. Here we show that a high-current ultrarelativistic electron beam interacting with multiple submicrometer-thick conducting foils can undergo strong self-focusing accompanied by efficient emission of gamma-ray synchrotron photons. Physically, self-focusing and high-energy photon emission originate from the beam interaction with the near-field transition radiation accompanying the beam-foil collision. This near field radiation is of amplitude comparable with the beam self-field, and can be strong enough that a single emitted photon can carry away a significant fraction of the emitting electron energy. After beam collision with multiple foils, femtosecond collimated electron and photon beams with number density exceeding that of a solid are obtained. The relative simplicity, unique properties, and high efficiency of this gamma-ray source open up new opportunities for both applied and fundamental research including laserless investigations of strong-field QED processes with a single electron beam.
\end{abstract}

DOI: 10.1103/PhysRevLett.126.064801

The generation of high-energy, dense, and collimated photon beams is of great interest both to fundamental and applied research. Indeed, such beams enable new avenues for research in strong-field QED, relativistic plasma astrophysics, and high-energy physics [1-4]. In particular, soliddensity photon beams allow matterless photon-photon physics studies, where traditional schemes are limited in luminosity due to the low density of high-energy photons [5]. A source of high-energy, solid-density photon beams also enables the generation of neutral collimated ultradense electron-positron jets, opening a unique portal to novel relativistic laboratory astrophysics studies [6-9]. Moreover, energetic solid-density electron and photon beams make it

Published by the American Physical Society under the terms of the Creative Commons Attribution 4.0 International license. Further distribution of this work must maintain attribution to the author(s) and the published article's title, journal citation, and DOI. Open access publication funded by the Max Planck Society. possible to access important unexplored regimes in highdensity beam physics $[10,11]$. Furthermore, intense sources of high-energy photons have broad applications in industry, medicine, and materials science [12-15].

The growing interest in intense high-energy photon sources has recently stimulated several proposals to further increase the attainable photon energy and flux. These proposals include high-power laser-plasma interactions [16-29], plasma instabilities [30], QED cascades [31,32], multiple colliding laser pulses [33,34], and beamstrahlung [35-37]. A number of experiments, where the generated photon beam properties could be accurately measured and tuned, were also successfully performed [38-45]. In those schemes, however, the achievable density remains less than $\sim 10^{24} \mathrm{~m}^{-3}$.

Here we introduce a novel concept for an ultraintense gamma-ray source based on the interaction of a single high-current ultrarelativistic electron beam with multiple submicrometer-thick conducting foils (see Fig. 1). By using fully 3D particle-in-cell (PIC) simulations, we show that (i) an ultrarelativistic $(10 \mathrm{GeV})$, dense 


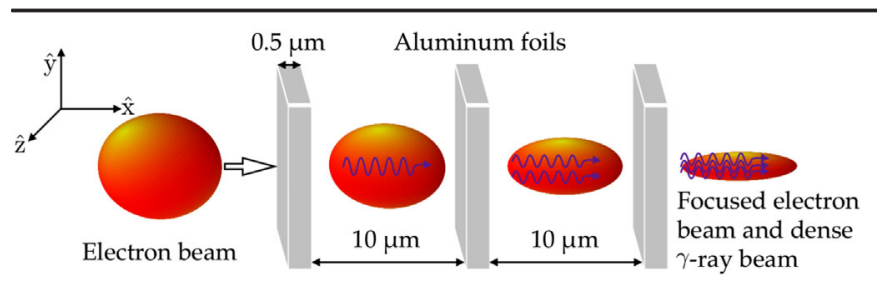

FIG. 1. Schematic setup. An ultrarelativistic electron beam sequentially collides with aluminum foils. At each beam-foil collision, a strong transverse force which focuses the electron beam and leads to copious gamma-ray emission is induced.

$\left(4.7 \times 10^{27} \mathrm{~m}^{-3}\right)$ electron beam can be radially focused up to $4.5 \times 10^{29} \mathrm{~m}^{-3}$, i.e., beyond the $1.8 \times 10^{29} \mathrm{~m}^{-3}$ electron density of solid aluminum; (ii) electron beam focusing is accompanied by intense synchrotron photon emission with more than $30 \%$ of the electron beam energy eventually converted into a $2.8 \times 10^{29} \mathrm{~m}^{-3}$ peak density collimated gamma-ray beam (with a maximum density of $8.5 \times 10^{29} \mathrm{~m}^{-3}$ achieved in the course of the interaction); (iii) when the electron beam density exceeds the foil electron density, the beam self-fields expel the target electrons and create an electron-depleted channel through the foil. The fields experienced by the beam electrons inside the plasma channel are so high that the quantum parameter $\chi \approx \gamma\left|f_{\perp}\right| / e F_{\text {cr }}$ exceeds unity [46,47]. Here $\boldsymbol{f}_{\perp}=q\left(\boldsymbol{E}_{\perp}+\boldsymbol{\beta} \times \boldsymbol{B}\right)$ is the Lorentz force transverse to the beam velocity, $\gamma$ the beam relativistic factor, $q=-e$ the electron charge, $F_{\mathrm{cr}}=m_{e}^{2} c^{3} / e \hbar \approx 1.3 \times 10^{18} \mathrm{~V} / \mathrm{m}$ the QED critical field $[4,46,47]$. This opens up the possibility of laserless strong-field QED investigations with only one ultrarelativistic electron beam [48].

We start by considering the free propagation of an electron beam in vacuum. The electric $\boldsymbol{E}$ and magnetic $\boldsymbol{B}$ self-fields of a cold electron beam in vacuum are related by $\boldsymbol{B}=\boldsymbol{\beta} \times \boldsymbol{E}$ [49], where $\boldsymbol{\beta}=\boldsymbol{v} / c$ is the normalized beam velocity (Gaussian units are employed for equations). Thus, $\boldsymbol{f}_{\perp}=q \boldsymbol{E}_{\perp} / \gamma^{2}$ is strongly suppressed for large $\gamma$, and the beam propagates almost ballistically over relatively long distances in vacuum.

When a beam collides with a conductor, it can be subject to strong near-field coherent transition radiation (CTR), which alters the nearly perfect cancellation of the electric and magnetic terms in the Lorentz force. Electromagnetic boundary conditions require that the electric field component tangential to the surface of a perfect conductor must be continuous and zero at the conductor surface, whereas the tangential magnetic field can be discontinuous and remains large [49]. Thus, when an electron beam encounters a conductor, the magnetic term of the Lorentz force, which drives beam focusing, can overcome the electric term, which drives beam expansion. Effectively, when the beam length is smaller than its transverse size, this process can be visualized as a beam colliding with its image charge (see below and Supplemental Material [50] for details on the near-field CTR fields, which include Refs. [51-56]). Notice that a large $f_{\perp}$ naturally results in intense emission of radiation. For instance, in the classical regime the radiated power (mean photon energy) is proportional to $\gamma^{2} f_{\perp}^{2}$ $\left(\gamma^{2} \boldsymbol{f}_{\perp}\right)[47,49]$.

For modeling, we consider an ultrarelativistic cold electron beam with cylindrical symmetry around its propagation axis $x$. The description is simplified by employing cylindrical coordinates with $r=\sqrt{y^{2}+z^{2}}$, $\theta=\arctan (z / y)$, and $x$ being the radial, azimuthal and vertical components, respectively. We assume that cylindrical symmetry is preserved throughout the interaction. Hence, fields are independent of $\theta$, the azimuthal electric field $E_{\theta}$ and the radial $B_{r}$ and vertical $B_{x}$ components of the magnetic field are zero. Here beam and conductor fields are denoted by the superscript $b$ and $c$, respectively.

For an ultrarelativistic charge distribution $\rho(x, r, t)=$ $\rho_{0} e^{-r^{2} / 2 \sigma_{\perp}^{2}} e^{-\left(x-x_{0}-v t\right)^{2} / 2 \sigma_{\|}^{2}}$ with $N_{e}$ electrons, initial position $x_{0}$, velocity $v$ along $x$, and peak charge density $\rho_{0}=q N_{e} /(2 \pi)^{3 / 2} \sigma_{\perp}^{2} \sigma_{\|}, E_{r}^{b} \gg E_{x}^{b} \approx 0$,

$E_{r}^{b}(x, r, t)=\frac{2 q N_{e}}{\sqrt{2 \pi} \sigma_{\|} r}\left(1-e^{-r^{2} / 2 \sigma_{\perp}^{2}}\right) e^{-\left(x-x_{0}-v t\right)^{2} / 2 \sigma_{\|}^{2}}$,

and $B_{\theta}^{b}(x, r, t)=\beta E_{r}^{b}(x, r, t)$, provide an approximate solution to Maxwell equations up to terms of order $1 / \gamma^{2}$ around the beam [48]. To evaluate $E_{r}^{c}(x, r, t)$ and $B_{\theta}^{c}(x, r, t)$, we consider a flat perfectly conducting foil with front surface at $x=0$. When the electron beam is outside the conductor, the method of images can be employed for determining $E_{r}^{c}(x, r, t)$ and $B_{\theta}^{c}(x, r, t)$ in $x<$ 0 [57]. This method cannot be applied when the beam enters the foil, because the image would be located in $x<0$, where conductor fields must satisfy source-free Maxwell equations. However, when $\sigma_{\perp} \gg \sigma_{\|}$one can approximate $E_{r}^{c}(x, r, t)$ and $B_{\theta}^{c}(x, r, t)$ with the image charge fields. This "reflected-field" approximation holds because CTR, which is emitted with transverse size $\sigma_{\perp}$ and typical wavelength $\sigma_{\|}$, undergoes weak diffraction over a Rayleigh length of approximately $\sigma_{\perp}^{2} / \sigma_{\|} \gg \sigma_{\|}$from the boundary. The opposite limit $\sigma_{\perp} \ll \sigma_{\|}$, corresponds to the magnetostatic approximation, yielding a vanishing $B_{\theta}^{c}$ and a surface-localized $E_{r}^{c}$ (see Supplemental Material [50]). Note that beam focusing in the $\sigma_{\perp} \ll \sigma_{\|}$limit has been demonstrated in accelerators [58-62].

The electron beam to radiated energy conversion efficiency $\eta$ can be calculated from $E_{r}=E_{r}^{b}+E_{r}^{c}$ and $B_{\theta}=B_{\theta}^{b}+B_{\theta}^{c}$, where Eq. (1) is employed for the beam and image charge fields. The average energy radiated per particle per unit time is conveniently approximated as [47] $\dot{\varepsilon}_{\gamma}=2 \alpha m_{e} c^{2} \chi^{2} / 3 \tau_{c}\left[1+4.8(1+\chi) \ln (1+1.7 \chi)+2.44 \chi^{2}\right]^{2 / 3}$, where $\alpha=e^{2} / \hbar c$ is the fine-structure constant, $\tau_{c}=$ $\hbar / m_{e} c^{2}$ is the Compton time, and $\chi \approx \gamma\left|E_{r}-B_{\theta}\right| / F_{\mathrm{cr}}$. Thus, 

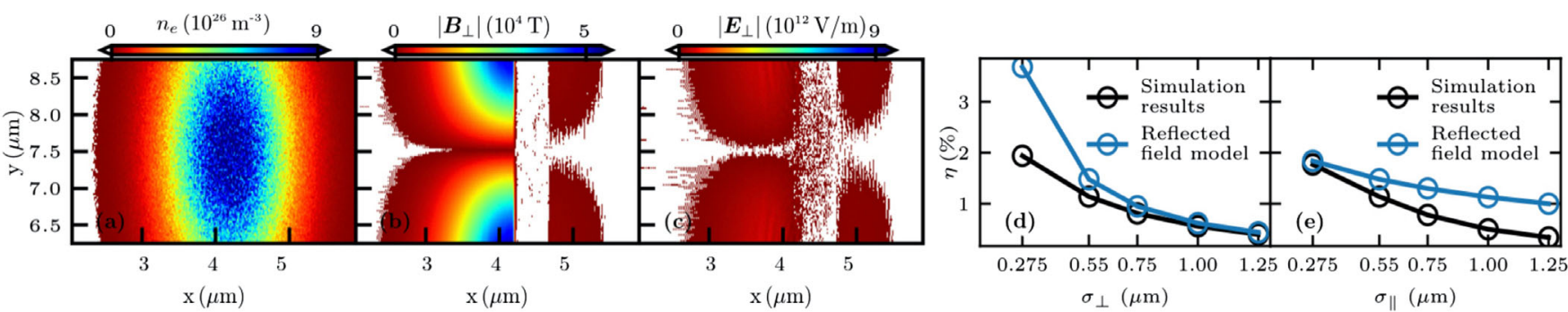

FIG. 2. (a) Electron beam density, (b) transverse magnetic field, and (c) transverse electric field in the collision with a $0.5 \mu$ m-thick aluminum foil. For comparison, the peak magnetic and electric beam self-fields are $3.1 \times 10^{4} \mathrm{~T}$, and $9.4 \times 10^{12} \mathrm{~V} / \mathrm{m}$, respectively. (d) Electron beam to radiation energy conversion efficiency $\eta$ as a function of $\sigma_{\perp}$ in the collision with one foil. The electron beam has $2 \mathrm{nC}$ charge, $10 \mathrm{GeV}$ energy, and $\sigma_{\|}=0.55 \mu \mathrm{m}$. Black circles: 3D PIC simulations results; blue circles: reflected-field model predictions. (e) Same as in panel (d) but for $\sigma_{\perp}=0.55 \mu \mathrm{m}$ and as a function of $\sigma_{\|}$.

$\eta=\frac{2 \pi \int_{-\infty}^{+\infty} d t \int_{-\infty}^{0} d x \int_{0}^{+\infty} d r r \rho(x, r, t) \dot{\varepsilon}_{\gamma}[\chi(x, r, t)]}{\gamma m_{e} c^{2} q N_{e}}$.

In Eq. (2) we have assumed that all electrons have the same initial momentum and energy $\gamma m_{e} c^{2}$. Furthermore, we have neglected the change in $\gamma$ during the beam-foil interaction. The triple integral in Eq. (2) can be carried out numerically.

Figure 2 shows the results of 3D PIC simulations of a cold electron beam colliding with one $0.5 \mu$ m-thick aluminum foil. The electron beam has $2 \mathrm{nC}$ charge, $10 \mathrm{GeV}$ energy, and Gaussian spatial distribution with $\sigma_{\|}=0.55 \mu \mathrm{m}, \sigma_{\perp}=1.25 \mu \mathrm{m}$, and $9.2 \times 10^{26} \mathrm{~m}^{-3}$ density. Figure 2(a) displays a snapshot of the electron beam density when the beam center has reached the front surface of the foil. Figures 2(b) and 2(c) show the transverse magnetic $\boldsymbol{B}_{\perp}$ and electric field $\boldsymbol{E}_{\perp}$, respectively. While $\boldsymbol{B}_{\perp}$ is amplified and its peak value nearly doubles with respect to the beam self-field $\left(3.1 \times 10^{4} \mathrm{~T}\right), \boldsymbol{E}_{\perp}$ is suppressed and much smaller than the beam self-field $\left(9.4 \times 10^{12} \mathrm{~V} / \mathrm{m}\right)$.

Figure 2(d) [Fig. 2(e)] plots $\eta$ during single electron beam-foil collision with the same parameters as above but for $\sigma_{\|}=0.55 \mu \mathrm{m}\left(\sigma_{\perp}=0.55 \mu \mathrm{m}\right)$ and $\sigma_{\perp}\left(\sigma_{\|}\right)$ranging from 0.275 to $1.25 \mu \mathrm{m}$. Black circles and blue circles correspond to 3D PIC simulation and reflected-field model results, respectively. These simulations confirm that the mechanism of beam focusing and photon emission is robust and effective. Indeed, as shown in the Supplemental Material [50], dense collimated photon beams can already be generated with the electron beam parameters attainable at existing accelerator facilities such as FACET-II [63].

Figures 2(d)-2(e) show that simulation results approach the prediction of the reflected-field model with increasing (decreasing) $\sigma_{\perp}\left(\sigma_{\|}\right)$. For beam density smaller than the foil electron density, simulations indicate that foil thickness is irrelevant provided that collisions and plasma instabilities remain negligible. By contrast, foil thickness is important when the electron beam density exceeds the conductor density [48]. Note that synchrotron photon emission also occurs when the beam exits the foil, as $E_{r}$ is suppressed at the rear foil surface and $B_{\theta}$ grows gradually during the beam exit [64]. However, for $\sigma_{\perp} \gtrsim \sigma_{\|}$, the contribution of the rear surface to the radiated energy is subdominant, and is neglected in our model.

The above considerations suggest that the focusing and radiative effects can be substantially enhanced by colliding the self-focused beam with further foils. In fact, the increased beam density results in stronger self-fields, thereby amplifying both self-focusing and photon emission at successive collisions (see Figs. 3-4). Note that, for efficient self-focusing, the distance between two consecutive foils needs to be sufficiently large to allow beam selffield restoration around its propagation axis $\left(r \lesssim \sigma_{\perp}\right)$. This requires that the travelled distance is much larger than $\sigma_{\perp}$. Furthermore, the interfoil distance needs to be short enough to prevent beam expansion. This can be estimated by considering the effect of $\left|\boldsymbol{f}_{\perp}\right| \approx\left|2 q B_{\theta}\right|$ calculated at $x \approx$ $x_{0}+v t$ and $r \approx \sigma_{\perp}$, i.e., where focusing is stronger. For $\sigma_{\perp} \gtrsim \sigma_{\|}$, CTR extends approximately over a distance $\sigma_{\perp}^{2} / \sigma_{\|}$, which is larger than the beam length $\sigma_{\|}$. Thus, $\left|\boldsymbol{f}_{\perp}\right|$ lasts for approximately $\sigma_{\|} / c$, and the deflection angle is $\vartheta \approx\left|f_{\perp}\right| \sigma_{\|} / \gamma m_{e} c^{2}$. Hence, to prevent defocusing the interfoil distance must be much smaller than $\sigma_{\perp} / \vartheta$. Also, for effective focusing, $\vartheta$ must be much larger than the beam angular spread $\Delta \vartheta_{p_{\perp} / p_{\|}} \approx \epsilon_{n} / \gamma \sigma_{\perp}$, where $\epsilon_{n}$ is the normalized beam emittance.

In our multifoil 3D PIC simulations, the electron beam has $2 \mathrm{nC}$ charge, Gaussian spatial and momentum distributions with $\sigma_{\|}=\sigma_{\perp}=0.55 \mu \mathrm{m}, 10 \mathrm{GeV}$ mean energy, $212 \mathrm{MeV}$ FWHM energy spread, and $3 \mathrm{~mm}$-mrad normalized emittance $(\vartheta \approx 2 \mathrm{mrad}$ with these parameters). Comparable parameters are expected at the advanced stage of FACET-II by employing a plasma lens $[63,65]$. The beam collides with 20 consecutive aluminum foils with $0.5 \mu \mathrm{m}$ thickness, $10 \mu \mathrm{m}$ interfoil distance, and $1.8 \times 10^{29} \mathrm{~m}^{-3}$ initial electron density. The computational box size is $6.6 \mu \mathrm{m}(x) \times 8.8 \mu \mathrm{m}(y) \times 8.8 \mu \mathrm{m}(z)$ with $528(x) \times 352(y) \times 352(z)$ grid points, 4 particles-per-cell (ppc) for beam electrons and $8 \mathrm{ppc}$ for foil electrons and 


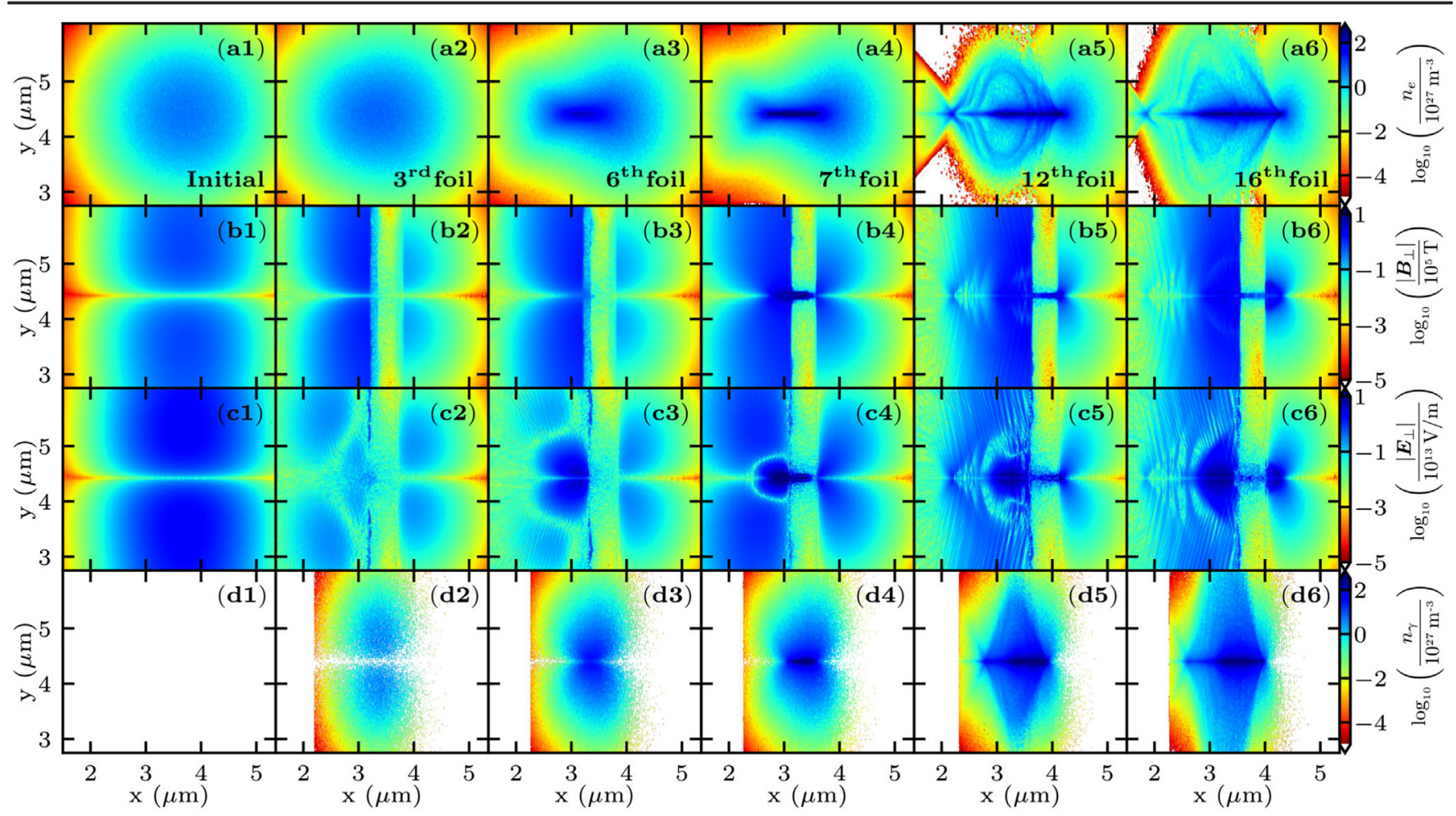

FIG. 3. Beam evolution. First column, initial electron beam density (a1), its magnetic (b1) and electric (c1) fields, and the initial photon density (d1). Second to sixth column, same quantities as in the first column but at the 3rd (a2)-(d2), the 6th (a3)-(d3), the 7th (a4)-(d4), the 12th (a5)-(d5), and the 16th (a6)-(d6) beam-foil interaction, respectively.

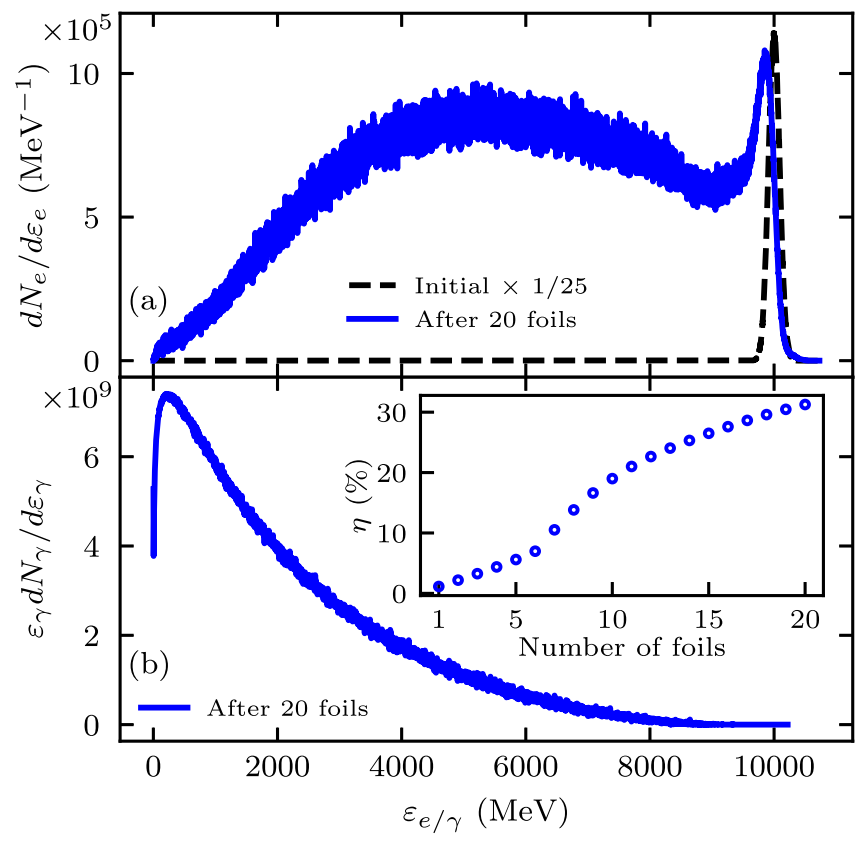

FIG. 4. (a) Initial (black dashed line) and final (blue line) electron beam energy distribution. (b) Final photon spectrum. The inset displays $\eta$ as a function of the number of foils crossed by the electron beam. ions were used. The moving window technique was employed to follow the beam evolution. Simulations were independently performed with Smilei $[66,67]$ and CALDER [68] PIC codes with good agreement. The initial self-consistent beam fields, the effect of field and collisional ionization and binary Coulomb collisions were included. Synchrotron and bremsstrahlung emission, and multiphoton Breit-Wheeler and Bethe-Heitler pair production were implemented with state-of-the-art Monte Carlo methods $[48,67,69,70]$. Consistent with the submicrometer foil thickness, simulations showed that collisional processes are negligible.

Figure 3 displays snapshots of the electron and gammaray beam evolution (see Supplemental Material [50] for a movie). Until the 6th foil, the beam interacts with the field "reflected" by each foil. This leads the beam to self-focus and gradually become denser (see the first to third column of Fig. 3). The electron beam density rises from its initial value of $4.7 \times 10^{27}$ to $8.2 \times 10^{28} \mathrm{~m}^{-3}$ after the 6 th foil, while the maximum photon density and $\chi$ are $2.9 \times 10^{28} \mathrm{~m}^{-3}$ and 0.8 , respectively [see Fig. 3(a3)$3(\mathrm{~d} 3)]$. During (immediately after) the interaction with the 7th foil, the electron beam density reaches $3.8 \times$ $10^{29} \mathrm{~m}^{-3}\left(4.5 \times 10^{29} \mathrm{~m}^{-3}\right)$, which exceeds the electron foil density of $1.8 \times 10^{29} \mathrm{~m}^{-3}$. Hence, the foil is unable to reflect the fields of the beam, and a channel where foil electrons are expelled is created [see Fig. 3(b4)-3(c4)]. 
Here $\chi$ and the photon beam density rise up to 3 and $4.1 \times 10^{29} \mathrm{~m}^{-3}$, respectively. The overall maximum gamma-ray density of $8.5 \times 10^{29} \mathrm{~m}^{-3}$ is reached immediately after electron beam interaction with the 8th foil. Moreover, a fraction of approximately $10^{-4}$ photons with energies $>2 m_{e} c^{2}$ convert into $e^{-} e^{+}$pairs via the multiphoton Breit-Wheeler process. Electron beam density stops increasing when it becomes larger than the foil electron density. In the following beam-foil collisions, the electron beam density profile undergoes longitudinal modulations, the reflected field strength being dependent on the longitudinal position and stronger around the rear part of the beam [Fig. 3(a5)-3(a6)].

Figure 4(a) plots the initial (black dashed line) and final (blue line) electron beam energy distribution after the interaction with 20 consecutive foils. The broad distribution around approximately $5 \mathrm{GeV}$ results from intense synchrotron emission occurring in the central and rear parts of the electron beam. The residual peak around the initial electron beam energy is indicative of the small synchrotron and collisional energy losses in the front part of the beam, which experiences only weak amplitude CTR. Figure 4(b) reports the final photon spectrum and the conversion efficiency $\eta$ (inset) as a function of the number of crossed foils. The increase in $\eta$ at the 7 th foil is due to the extremely high beam density and, consequently, to the ultrastrong fields induced inside the foil. After colliding with 20 foils, more than $30 \%$ of the electron beam energy is converted into a collimated $(5 \mathrm{mrad} \mathrm{rms}$ photon energy angular distribution), 4 fs FWHM duration, $2.8 \times 10^{29} \mathrm{~m}^{-3}$ peak density gamma-ray pulse.

In summary, we have introduced a new scheme to efficiently produce extremely dense gamma-ray beams from the interaction of a high-current ultrarelativistic electron beam with a sequence of thin foils. This scheme also provides a promising route for producing solid-density ultrarelativistic electron beams and for exploring strongfield QED processes with a single electron beam, that is, without the need of an external powerful laser drive. In fact, following a methodology analogous to that employed with intense laser pulses [43-45], the field experienced in situ by the electron beam and the ensuing strong-field QED effects can be inferred by measuring particle angular distributions, spectra, and photon and pair yields along with CTR [71-74].

This work was performed in the framework of the E-305 Collaboration. E-305 is a SLAC experiment whose aims include the generation of bright gamma rays, in particular, in electron beam-solid interaction. Based on the findings of this work, the E-332 experiment on solid-density gammaray pulse generation in electron beam-multifoil interaction has been developed and approved, and will be carried out at SLAC. This article comprises parts of the Ph.D. thesis work of Archana Sampath, submitted to the Heidelberg University, Germany. The work at LOA was supported by the European Research Council (ERC) under the European Unions Horizon 2020 research and innovation programme (Grant Agreement No. 715807). We acknowledge GENCI for granting us access to the supercomputer Irene under the Grant No. A0080510786 to run CALDER simulations. The work at SLAC was supported by U.S. DOE FES Grant No. FWP100331. UCLA was supported by U.S. Department of Energy Grant No. DE-SC001006 and NSF Grant No. 1734315.

*matteo.tamburini@mpi-hd.mpg.de

[1] G. A. Mourou, T. Tajima, and S. V. Bulanov, Optics in the relativistic regime, Rev. Mod. Phys. 78, 309 (2006).

[2] M. Marklund and P. K. Shukla, Nonlinear collective effects in photon-photon and photon-plasma interactions, Rev. Mod. Phys. 78, 591 (2006).

[3] R. Ruffini, G. Vereshchagin, and S.-S. Xue, Electronpositron pairs in physics and astrophysics: From heavy nuclei to black holes, Phys. Rep. 487, 1 (2010).

[4] A. Di Piazza, C. Müller, K. Z. Hatsagortsyan, and C. H. Keitel, Extremely high-intensity laser interactions with fundamental quantum systems, Rev. Mod. Phys. 84, 1177 (2012).

[5] V. I. Telnov, Problems in obtaining $\gamma \gamma$ and $\gamma e$ colliding beams at linear colliders, Nucl. Instrum. Methods Phys. Res., Sect. A 294, 72 (1990).

[6] G. Sarri et al., Generation of neutral and high-density electron-positron pair plasmas in the laboratory, Nat. Commun. 6, 6747 (2015).

[7] H. Chen, F. Fiuza, A. Link, A. Hazi, M. Hill, D. Hoarty, S. James, S. Kerr, D. D. Meyerhofer, J. Myatt, J. Park, Y. Sentoku, and G. J. Williams, Scaling the Yield of LaserDriven Electron-Positron Jets to Laboratory Astrophysical Applications, Phys. Rev. Lett. 114, 215001 (2015).

[8] M. Lobet, C. Ruyer, A. Debayle, E. d'Humières, M. Grech, M. Lemoine, and L. Gremillet, Ultrafast SynchrotronEnhanced Thermalization of Laser-Driven Colliding Pair Plasmas, Phys. Rev. Lett. 115, 215003 (2015).

[9] C. D. Arrowsmith, N. Shukla, N. Charitonidis, R. Boni, H. Chen, T. Davenne, D. H. Froula, B. T. Huffman, Y. Kadi, B. Reville, S. Richardson, S. Sarkar, J. L. Shaw, L. O. Silva, R. M. G. M. Trines, R. Bingham, and G. Gregori, Generating ultra-dense pair beams using $400 \mathrm{GeV} / \mathrm{c}$ protons, arXiv:2011.04398.

[10] F. Del Gaudio, R. Fonseca, L. O. Silva, and T. Grismayer, Plasma Wakes Driven by Photon Bursts Via Compton Scattering, Phys. Rev. Lett. 125, 265001 (2020).

[11] X. Xu, D. B. Cesar, S. Corde, V. Yakimenko, M. J. Hogan, C. Joshi, A. Marinelli, and W. B. Mori, Generation of terawatt, attosecond pulses from relativistic transition radiation, arXiv:2007.12736.

[12] D. H. Bilderback, P. Elleaume, and E. Weckert, Review of third and next generation synchrotron light sources, J. Phys. B 38, S773 (2005).

[13] J. Ullrich, A. Rudenko, and R. Moshammer, Free-electron lasers: New avenues in molecular physics and photochemistry, Annu. Rev. Phys. Chem. 63, 635 (2012). 
[14] S. Corde, K. T. Phuoc, G. Lambert, R. Fitour, V. Malka, A. Rousse, A. Beck, and E. Lefebvre, Femtosecond x rays from laser-plasma accelerators, Rev. Mod. Phys. 85, 1 (2013).

[15] http://www.lightsources.org/.

[16] C. P. Ridgers, C. S. Brady, R. Duclous, J. G. Kirk, K. Bennett, T. D. Arber, A. P. L. Robinson, and A. R. Bell, Dense Electron-Positron Plasmas and Ultraintense $\gamma$ Rays from Laser-Irradiated Solids, Phys. Rev. Lett. 108, 165006 (2012).

[17] T. Nakamura, J. K. Koga, T. Z. Esirkepov, M. Kando, G. Korn, and S. V. Bulanov, High-Power $\gamma$-Ray Flash Generation in Ultraintense Laser-Plasma Interactions, Phys. Rev. Lett. 108, 195001 (2012).

[18] L. L. Ji, A. Pukhov, E. N. Nerush, I. Yu. Kostyukov, B. F. Shen, and K. U. Akli, Energy partition, $\gamma$-ray emission, and radiation reaction in the near-quantum electrodynamical regime of laser-plasma interaction, Phys. Plasmas 21, 023109 (2014).

[19] J.-X. Li, K. Z. Hatsagortsyan, B. J. Galow, and C. H. Keitel, Attosecond Gamma-Ray Pulses Via Nonlinear Compton Scattering in the Radiation-Dominated Regime, Phys. Rev. Lett. 115, 204801 (2015).

[20] X.-L. Zhu, Y. Yin, T.-P. Yu, F.-Q. Shao, Z.-Y. Ge, W.-Q. Wang, and J.-J. Liu, Enhanced electron trapping and $\gamma$ ray emission by ultra-intense laser irradiating a near-criticaldensity plasma filled gold cone, New J. Phys. 17, 053039 (2015).

[21] D. J. Stark, T. Toncian, and A. V. Arefiev, Enhanced Multi$\mathrm{MeV}$ Photon Emission by a Laser-Driven Electron Beam in a Self-Generated Magnetic Field, Phys. Rev. Lett. 116, 185003 (2016).

[22] H. X. Chang, B. Qiao, T. W. Huang, Z. Xu, C. T. Zhou, Y. Q. Gu, X. Q. Yan, M. Zepf, and X. T. He, Brilliant petawatt gamma-ray pulse generation in quantum electrodynamic laser-plasma interaction, Sci. Rep. 7, 45031 (2017).

[23] W.-M. Wang, Z.-M. Sheng, P. Gibbon, L.-M. Chen, Yu.-T. $\mathrm{Li}$, and J. Zhang, Collimated ultrabright gamma rays from electron wiggling along a petawatt laser-irradiated wire in the QED regime, Proc. Natl. Acad. Sci. U.S.A. 115, 9911 (2018).

[24] T. W. Huang, C. M. Kim, C. T. Zhou, C. M. Ryu, K. Nakajima, S.C. Ruan, and C. H. Nam, Tabletop laserdriven gamma-ray source with nanostructured doublelayer target, Plasma Phys. Controlled Fusion 60, 115006 (2018).

[25] T. W. Huang, C. M. Kim, C. T. Zhou, M. H. Cho, K. Nakajima, C. M. Ryu, S. C. Ruan, and C. H. Nam, Highly efficient laser-driven Compton gamma-ray source, New J. Phys. 21, 013008 (2019).

[26] M. Vranic, T. Grismayer, S. Meuren, R. A. Fonseca, and L. O. Silva, Are we ready to transfer optical light to gammarays?, Phys. Plasmas 26, 053103 (2019).

[27] M. Jirka, O. Klimo, Y.-J. Gu, and S. Weber, Enhanced photon emission from a double-layer target at moderate laser intensities, Sci. Rep. 10, 8887 (2020).

[28] J. Ferri, S. Corde, A. Döpp, A. Lifschitz, A. Doche, C. Thaury, K. Ta Phuoc, B. Mahieu, I. A. Andriyash, V. Malka, and X. Davoine, High-Brilliance Betatron $\gamma$-Ray Source Powered by Laser-Accelerated Electrons, Phys. Rev. Lett. 120, 254802 (2018).
[29] X.-L. Zhu, M. Chen, S.-M. Weng, T.-P. Yu, W.-M. Wang, F. He, Z.-M. Sheng, P. McKenna, D. A. Jaroszynski, and J. Zhang, Extremely brilliant $\mathrm{GeV} \gamma$-rays from a two-stage laser-plasma accelerator, Sci. Adv. 6, eaaz7240 (2020).

[30] A. Benedetti, M. Tamburini, and C. H. Keitel, Giant collimated gamma-ray flashes, Nat. Photonics 12, 319 (2018).

[31] M. Jirka, O. Klimo, S. V. Bulanov, T. Z. Esirkepov, E. Gelfer, S. S. Bulanov, S. Weber, and G. Korn, Electron dynamics and $\gamma$ and $e^{-} e^{+}$production by colliding laser pulses, Phys. Rev. E 93, 023207 (2016).

[32] M. Tamburini, A. Di Piazza, and C. H. Keitel, Laser-pulseshape control of seeded QED cascades, Sci. Rep. 7, 5694 (2017).

[33] A. Gonoskov, A. Bashinov, S. Bastrakov, E. Efimenko, A. Ilderton, A. Kim, M. Marklund, I. Meyerov, A. Muraviev, and A. Sergeev, Ultrabright GeV Photon Source Via Controlled Electromagnetic Cascades in Laser-Dipole Waves, Phys. Rev. X 7, 041003 (2017).

[34] J. Magnusson, A. Gonoskov, M. Marklund, T. Z. Esirkepov, J. K. Koga, K. Kondo, M. Kando, S. V. Bulanov, G. Korn, and S. S. Bulanov, Laser-Particle Collider for Multi-GeV Photon Production, Phys. Rev. Lett. 122, 254801 (2019).

[35] V. Yakimenko, S. Meuren, F. Del Gaudio, C. Baumann, A. Fedotov, F. Fiuza, T. Grismayer, M. J. Hogan, A. Pukhov, L. O. Silva, and G. White, Prospect of Studying Nonperturbative QED with Beam-Beam Collisions, Phys. Rev. Lett. 122, 190404 (2019).

[36] F. Del Gaudio, T. Grismayer, R. A. Fonseca, W. B. Mori, and L. O. Silva, Bright $\gamma$ rays source and nonlinear BreitWheeler pairs in the collision of high density particle beams, Phys. Rev. Accel. Beams 22, 023402 (2019).

[37] M. Tamburini and S. Meuren, Efficient High-Energy Photon Production in the supercritical QED regime, arXiv:1912.07508.

[38] H.-P. Schlenvoigt, K. Haupt, A. Debus, F. Budde, O. Jäckel, S. Pfotenhauer, H. Schwoerer, E. Rohwer, J. G. Gallacher, E. Brunetti, R. P. Shanks, S. M. Wiggins, and D. A. Jaroszynski, A compact synchrotron radiation source driven by a laser-plasma wakefield accelerator, Nat. Phys. 4, 130 (2008).

[39] S. Kneip et al., Bright spatially coherent synchrotron x-rays from a table-top source, Nat. Phys. 6, 980 (2010).

[40] S. Cipiccia et al., Gamma-rays from harmonically resonant betatron oscillations in a plasma wake, Nat. Phys. 7, 867 (2011).

[41] K. T. Phuoc, S. Corde, C. Thaury, V. Malka, A. Tafzi, J. P. Goddet, R. C. Shah, S. Sebban, and A. Rousse, Alloptical Compton gamma-ray source, Nat. Photonics 6, 308 (2012)

[42] G. Sarri, D. J. Corvan, W. Schumaker, J. M. Cole, A. Di Piazza, H. Ahmed, C. Harvey, C. H. Keitel, K. Krushelnick, S. P. D. Mangles, Z. Najmudin, D. Symes, A. G. R. Thomas, M. Yeung, Z. Zhao, and M. Zepf, Ultrahigh Brilliance Multi-MeV $\gamma$-Ray Beams from Nonlinear Relativistic Thomson Scattering, Phys. Rev. Lett. 113, 224801 (2014).

[43] W. Yan, C. Fruhling, G. Golovin, D. Haden, J. Luo, P. Zhang, B. Zhao, J. Zhang, C. Liu, M. Chen, S. Chen, S. Banerjee, and D. Umstadter, High-order multiphoton Thomson scattering, Nat. Photonics 11, 514 (2017). 
[44] J. M. Cole et al., Experimental Evidence of Radiation Reaction in the Collision of a High-Intensity Laser Pulse with a Laser-Wakefield Accelerated Electron Beam, Phys. Rev. X 8, 011020 (2018).

[45] K. Poder et al., Experimental Signatures of the Quantum Nature of Radiation Reaction in the Field of an Ultraintense Laser, Phys. Rev. X 8, 031004 (2018).

[46] V. I. Ritus, Quantum effects of the interaction of elementary particles with an intense electromagnetic field, J. Russ. Laser Res. 6, 497 (1985).

[47] V. N. Baier, V. M. Katkov, and V. M. Strakhovenko, Electromagnetic Processes at High Energies in Oriented Single Crystals (World Scientific, Singapore, 1998).

[48] A. Sampath, Ph.D. thesis, Ruprecht-Karls-Universität, Heidelberg, 2020.

[49] J. D. Jackson, Classical Electrodynamics, 3rd ed. (John Wiley and Sons, Inc., 1998).

[50] See Supplemental Material at http://link.aps.org/ supplemental/10.1103/PhysRevLett.126.064801 for (i) modeling of the near-field CTR; (ii) PIC simulations at presently achievable beam densities; (iii) a movie of the electron and gamma beam evolution in the interaction with 20 consecutive foils.

[51] V. Ginzburg and V. N. Tsytovich, Transition Radiation and Transition Scattering (Adam Hilger, Bristol, UK, 1990).

[52] I. S. Gradshteyn, I. M. Ryzhik, A. Jeffrey, and D. Zwillinger, Table of Integrals, Series, and Products (Academic Press, Burlington, USA, 2007).

[53] V. A. Verzilov, Transition radiation in the pre-wave zone, Phys. Lett. A 273, 135 (2000).

[54] J. E. Harvey, Fourier treatment of near-field scalar diffraction theory, Am.J. Phys. 47, 974 (1979).

[55] M. Castellano and V.A. Verzilov, Spatial resolution in optical transition radiation beam diagnostics, Phys. Rev. ST Accel. Beams 1, 062801 (1998).

[56] A. F. Lifschitz, X. Davoine, E. Lefebvre, J. Faure, C. Rechatin, and V. Malka, Particle-in-cell modelling of laser-plasma interaction using fourier decomposition, J. Comput. Phys. 228, 1803 (2009).

[57] P. Hammond, Electric and magnetic images, Proc IEE Part C Monographs 107, 306 (1960).

[58] R. J. Adler, Image-field focusing of intense ultra-relativistic electron beams in vacuum, Part. Accel. 12, 39 (1982), http:// cdsweb.cern.ch/record/1107997/files/.

[59] S. Humphries, Equilibria for foil-focused relativistic electron beams, Part. Accel. 13, 249 (1983), http://cds.cern.ch/ record/1108004/files/.

[60] S. Humphries and C. B. Ekdahl, Image charge focusing of relativistic electron beams, J. Appl. Phys. 63, 583 (1988).

[61] S. Humphries, C. Ekdahl, and D. M. Woodall, Image current guiding of a relativistic electron beam in a foil focusing system, Appl. Phys. Lett. 54, 2195 (1989).

[62] R. F. Fernsler, R. F. Hubbard, and S. P. Slinker, Foil focusing of electron beams, J. Appl. Phys. 68, 5985 (1990).
[63] V. Yakimenko, L. Alsberg, E. Bong, G. Bouchard, C. Clarke, C. Emma, S. Green, C. Hast, M. J. Hogan, J. Seabury, N. Lipkowitz, B. O'Shea, D. Storey, G. White, and G. Yocky, FACET-II facility for advanced accelerator experimental tests, Phys. Rev. Accel. Beams 22, 101301 (2019).

[64] N. J. Carron, Fields of particles and beams exiting a conductor, Prog. Electromagn. Res. 28, 147 (2000).

[65] C. E. Doss, E. Adli, R. Ariniello, J. Cary, S. Corde, B. Hidding, M. J. Hogan, K. Hunt-Stone, C. Joshi, K. A. Marsh, J. B. Rosenzweig, N. Vafaei-Najafabadi, V. Yakimenko, and M. Litos, Laser-ionized, beam-driven, underdense, passive thin plasma lens, Phys. Rev. Accel. Beams 22, 111001 (2019).

[66] J. Derouillat, A. Beck, F. Prez, T. Vinci, M. Chiaramello, A. Grassi, M. Fl, G. Bouchard, I. Plotnikov, N. Aunai, J. Dargent, C. Riconda, and M. Grech, Smilei: A collaborative, open-source, multi-purpose particle-in-cell code for plasma simulation, Comput. Phys. Commun. 222, 351 (2018).

[67] Smilei particle-in-cell code, https://smileipic.github.io/ Smilei/.

[68] E. Lefebvre et al., Electron and photon production from relativistic laser-plasma interactions, Nucl. Fusion 43, 629 (2003).

[69] M. Lobet, E. d'Humières, M. Grech, C. Ruyer, X. Davoine, and L. Gremillet, Modeling of radiative and quantum electrodynamics effects in PIC simulations of ultrarelativistic laser-plasma interaction, J. Phys. Conf. Ser. 688, 012058 (2016).

[70] B. Martinez, M. Lobet, R. Duclous, E. d'Humières, and L. Gremillet, High-energy radiation and pair production by Coulomb processes in particle-in-cell simulations, Phys. Plasmas 26, 103109 (2019).

[71] J. van Tilborg, C. B. Schroeder, C. V. Filip, C. Tóth, C. G. R. Geddes, G. Fubiani, R. Huber, R. A. Kaindl, E. Esarey, and W. P. Leemans, Temporal Characterization of Femtosecond Laser-Plasma-Accelerated Electron Bunches Using Terahertz Radiation, Phys. Rev. Lett. 96, 014801 (2006).

[72] Y. Glinec, J. Faure, A. Norlin, A. Pukhov, and V. Malka, Observation of Fine Structures in Laser-Driven Electron Beams Using Coherent Transition Radiation, Phys. Rev. Lett. 98, 194801 (2007).

[73] T. J. Maxwell, C. Behrens, Y. Ding, A. S. Fisher, J. Frisch, Z. Huang, and H. Loos, Coherent-Radiation Spectroscopy of Few-Femtosecond Electron Bunches Using a MiddleInfrared Prism Spectrometer, Phys. Rev. Lett. 111, 184801 (2013).

[74] O. Lundh, C. Rechatin, J. Lim, V. Malka, and J. Faure, Experimental Measurements of Electron-Bunch Trains in a Laser-Plasma Accelerator, Phys. Rev. Lett. 110, 065005 (2013). 